\title{
Calibration of Vibration Pickups by the Reciprocity Method
}

\author{
Samuel Levy and Raymond R. Bouche
}

\begin{abstract}
The reciprocity theory for the relationship between mechanical force and velocity and electric current and voltage is presented for a linear electrodynamic vibration-pickup calibrator having a driving coil, a velocity-sensing coil, and a mounting table. The theory takes account of flexibility in the calibrator structure and in the coils, electric coupling between different parts of the same coil and between parts of one coil and parts of the other, and flexibility in the magnet structure. The theory shows what measurements are required in using a linear electrodynamic calibrator for the absolute calibration of vibration pickups.

A description is given of the mechanical arrangement and electric circuitry used at the National Bureau of Standards in calibrating calibrators by the reciprocity method. A typical velocity-sensing-coil calibration curve is presented showing the effect of pickup mass on the calibration factor. Typical examples of measurement of calibration factor and mechanical impedance of pickups are also presented.

Practical limitations of the reciprocity method due to nonlinearity resulting from lack of tightness of mechanical joints, resonance effects, amplitude effects, etc., are discussed.
\end{abstract}

\section{Introduction}

An early application of the reciprocity method to the measurement of mechanical quantities was that by R. K. Cook [1] ${ }^{1}$ in 1940. He applied the method to the absolute calibration of microphones. This was followed in 1948 with papers by H. M. Trent [2] and A. London [3], who showed how the reciprocity method could be used for the absolute calibration of vibration pickups. The reciprocity method was applied to the calibration of electrodynamic transducers in 1948 by S. P. Thompson [4] and to the calibration of piezoelectric accelerometers in 1952 by M. Harrison, A. O. Sykes, and P. G. Marcotte [5].

Commercially available calibrators for vibration pickups have a mounting table that is connected by a relatively rigid internal structure to a driving coil and to a velocity-sensing coil. This internal structure is supported from the frame by flexure springs or guide wires. John C. Camm, formerly of NBS, in 1953 showed how the reciprocity method could be applied to the calibration of the velocity-sensing coil of such a calibrator in connection with work for the Office of Naval Research. In 1955 the authors of the present report, in connection with work for the Diamond Ordnance Fuze Laboratories, extended Camm's theory to take account of pickup mass and flexibility in the internal structure for the frequently occurring case where the driving coil, sensing coil, and mounting table are mechanically joined at a point.

The present report gives a broader basis for the application of the reciprocity method to the calibration of the velocity-sensing coil of calibrators, by considering both the internal structure and the magnet to be flexible and by taking account of electric-coupling effects. It also shows how the calibrator, once its velocity-sensing coil has been calibrated by the reciprocity method, can be used to determine the calibration factor and to measure the mechanical impedance of vibration pickups.

\section{Reciprocity Theory for Flexible Vibration-Pickup Calibrators}

In the appendix the general reciprocity theory for a calibrator is presented. This theory is applicable to a calibrator operating in any frequency or amplitude range in which it can be considered to be a linear system. Neither the internal structure nor the magnet structure need be considered rigid. The theory is applicable, for example, not only at low frequencies and at frequencies near certain resonances, but also at frequencies above axial resonance where

${ }^{1}$ Figures in brackets indicate the literature references at the end of this paper. 
the driving coil and mounting table move in opposite directions. The theory is also applicable where there is electric coupling between different parts of the same coil and between parts of one coil and parts of the other.

The positive terminals of the driving and sensing coils are designated as those having positive voltages when the mounting table is moving inward. Conversely, positive current in the coils produces outward velocity at the mounting table.

When the calibrator is energized with current in the driving coil at any particular frequency, the calibration factor, $F$, is defined as the ratio of the voltage in volts in the velocitysensing coil to the velocity in inches per second at the surface of the mounting table. It is shown in the appendix that

$$
F=a+b Y_{p}
$$

where $Y_{p}$ is the pickup mechanical impedance (lb-sec/in.), and all the symbols represent complex numbers in the manner commonly used for alternating-current electrical theory. The calibrator constants, $a$ and $b$, are determined by the following two experiments and computational procedure.

Experiment 1: The equipment is shown in figure 1 (a). Attach weights to the mounting table and measure for each weight the transfer admittance in amperes per volt, $G$, between the driving and sensing coils:

$$
G=\frac{I_{o}^{D}}{E_{o}^{S}}
$$

where

$I_{o}^{D}=$ current in driving coil.

$E_{0}^{S}=$ voltage generated in open-circuited sensing coil. (It is permissible to leave a voltmeter permanently connected to the sensing coil. If this is done, the open-circuit condition is obtained when no other connection is made to the sensing coil.)

Experiment 2: The equipment is shown in figure 1 (b). Couple a second vibration exciter to the calibrator being calibrated at the mounting table, and measure the ratio, $R$, of opencircuited voltages generated in the sensing and driving coils:

$$
R=E_{o}^{S} / E_{o}^{D} .
$$

Computational Procedure: Determine the ordinate intercept, $J$, and the slope, $Q$, of the function $W /\left(G-G_{0}\right)$ when plotted against the weight, $W$, attached to the mounting table in experiment 1 , where $G_{o}$ is the value of $G$ when $W=0$. Constants $a$ and $b$ in eq (1) are then given by

$$
a=0.01711 \sqrt{j \omega J R}, \quad b=6.601 Q \sqrt{R /(j \omega J)},
$$

where $\omega$ is the frequency in radians per second, and $j$ is the unit imaginary vector.

It is shown in the appendix that the vibration-pickup calibrator calibrated by the reciprocity method can be used to determine the mechanical impedance, $Y_{p}$, of a pickup by measuring the admittance, $G_{p}$, eq (2), with the pickup attached to the mounting table, and the admittance, $G_{o}$, when nothing is attached to the table, by use of the relationship

$$
Y_{p}=j\left(\frac{\omega}{386}\right)\left(\frac{J\left(G_{p}-G_{o}\right)}{1-Q\left(G_{p}-G_{o}\right)}\right)
$$

To carry out the calibration of a pickup the following procedure is used: The driving coil of the calibrator is energized at the desired frequency and the value of $Y_{p}$ is determined. The 

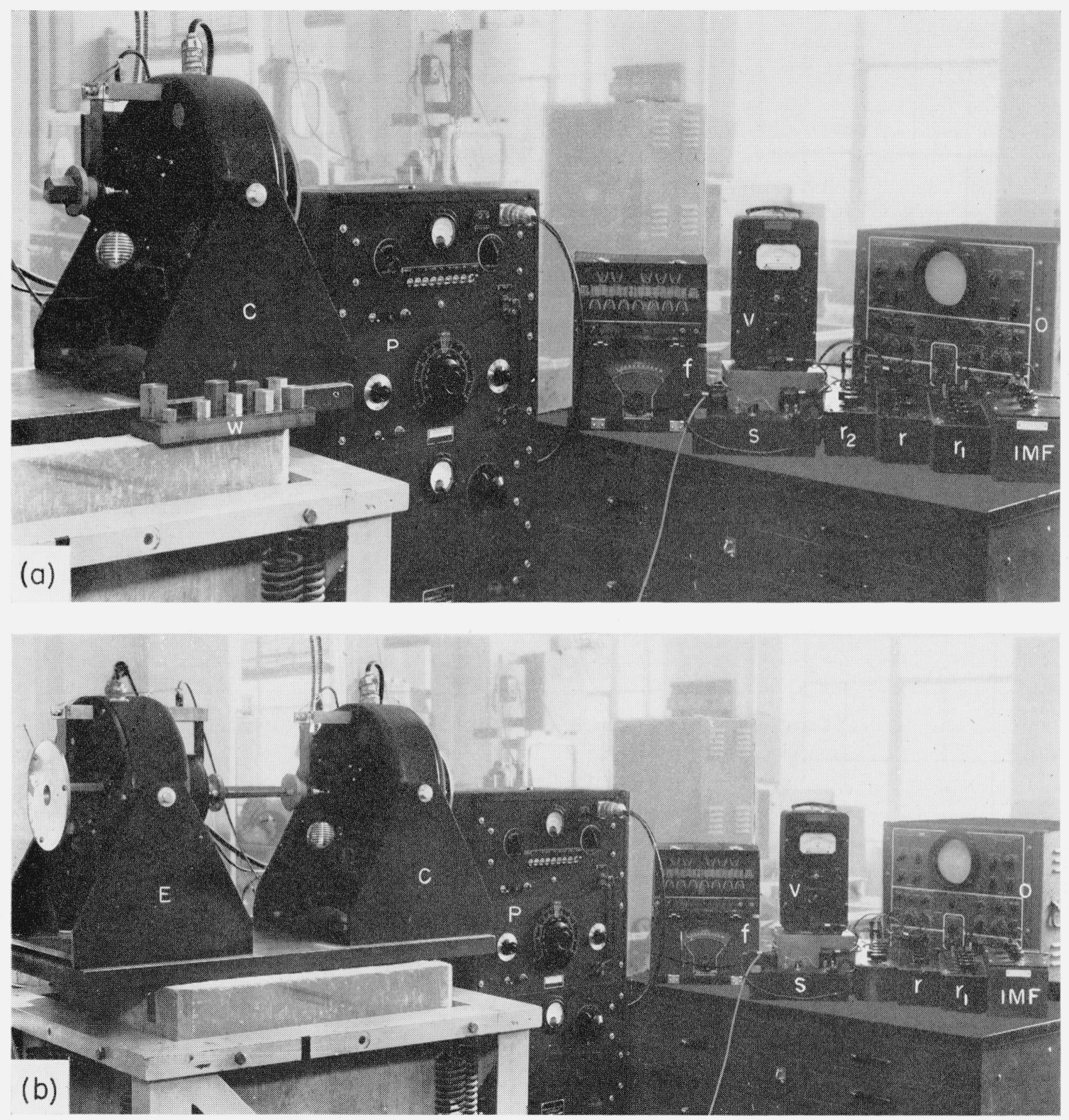

FiguRE 1. Measurement of constants of vibration-pickup calibrator.

(a) Weights, $W$, are attached to mounting table of calibrator, $C$, in experiment 1. (b) External vibration exciter, $E$, is required to provide sinusoidal motion to calibrator, $C$, in experiment 2 .

calibrator is then driven at the desired amplitude as indicated by the voltage, $E_{o}^{S}$, in the sensing coil, and then the velocity is given by

$$
v=E_{o}^{S} / F,
$$

where $F$ is obtained by substituting $Y_{p}$ in eq (1). The output of the pickup corresponding to this excitation is then measured. Ordinarily the term $b Y_{p}$ in eq (1) for $F$ is negligibly small, except at frequencies high enough to cause relative displacements between the mounting table and the sensing coil. Where it is known that $b Y_{p}$ is negligibly small, the determination of $Y_{p}$ may be omitted from the calibration procedure. 


\section{Measurement of Constants}

\subsection{Accuracy of Weights}

The weights attached to the table in carrying out experiment 1 at the Bureau are shown at $W$ in figure 1 (a). The weights increase in 0.1 -lb steps from 0 to $1 \mathrm{lb}$. They vary less than 0.1 percent from their rated values. Their attachment surface has a stud that engages the mounting table and a contacting ring, $1 / 8$ in. wide, which provides a connection of high rigidity. A film of oil is wiped on this ring before engagement to eliminate air in the contact surface.

\subsection{Accuracy of Frequency Measurements}

The frequency was measured with a calibrated electronic frequency meter. The indicated audio oscillator frequency did not differ from the measured frequency by more than 0.2 percent.

\subsection{Measurement of Transfer Admittance}

The circuit shown in figure 2 (a) is used to measure the transfer admittance. With the switch in the "up" position, the values of $r$ and $r_{1}$ are adjusted until the voltage drops $\left|E_{12}\right|$ across terminals 1 and 2 , and $\left|E_{13}\right|$ across terminals 1 and 3 , are equal as measured on a highimpedance voltmeter. The magnitude of $G$ is then given by

$$
|G|=\frac{r+r_{1}+r_{2}}{r r_{2}}
$$

The accuracy of $|G|$ determined in this manner depends only on the accuracy of the circuit elements and the repeatability of the voltmeter reading, but not on the accuracy of the voltmeter. Values of the resistances are chosen to load the amplifier suitably. Typical values for this calibrator are $r_{2}$, equal to $10 \mathrm{ohms}$, and $r+r_{1}$, approximately 1,000 times greater than $r_{2}$.

Voltage $\left|E_{23}\right|$ across terminals 2 and 3 is then read from the voltmeter. The switch is lowered to the "down" position. The value of $r_{1}$ is adjusted until $\left|E_{14}\right|$ equals $\left|E_{13}\right|$, and voltages $\left|E_{13}\right|$ and $\left|E_{43}\right|$ are read on the voltmeter. The phase angle, $\phi_{G}$, of the transfer admittance, $G$, is the phase angle of $I_{o}^{D}$ with respect to $E_{o}^{S}$. Because the currents in $r, r_{1}$, and $r_{2}$ are in phase with $I_{o}^{D}$, phase angle $\phi_{G}$ can be determined from a construction of a polygon of voltages as

$$
\phi_{G}=\cos ^{-1}\left(1-\frac{1}{2} \frac{\left|E_{23}^{2}\right|}{\left|E_{13}^{2}\right|}\right) .
$$

A suitable value of $r_{2}$ is used so that $r_{1} \omega 10^{-6}$ is greater than 100 ; then the approximate value of
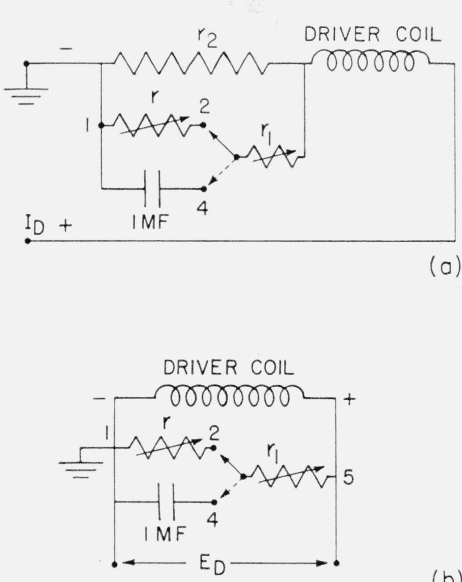

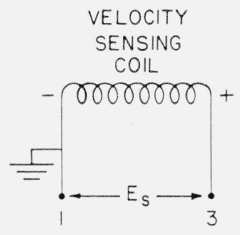

Figure 2. Circuits used in the measurement of constants of vibration-pickup calibrator.

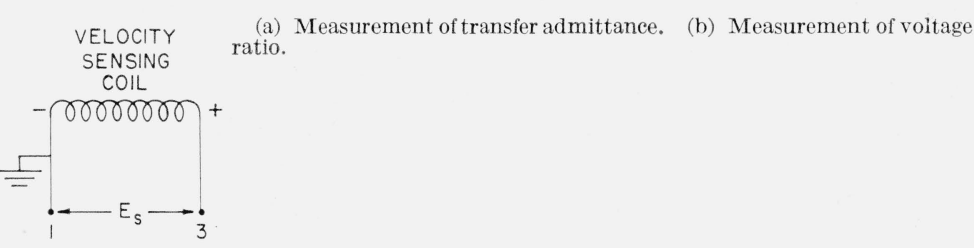


$\phi_{G}$ can be determined from

$$
\left(\phi_{G}\right) \text { approx }=90^{\circ}-\cos ^{-1}\left(1-\frac{1}{2} \frac{\left|E_{34}^{2}\right|}{\left|E_{13}^{2}\right|}\right) .
$$

The use of both eq (8) and (9) determines the magnitude and quadrant for $\phi_{G}$. It happens, though, except near certain resonances, that $\phi_{G}$ is near $\pm 90^{\circ}$. Small errors in $\left|E_{43}\right|$ or $\left|E_{13}\right|$ result in large changes in the angle

$$
\cos ^{-1}\left(1-\frac{1}{2} \frac{\left|E_{43}^{2}\right|}{\left|E_{13}^{2}\right|}\right)
$$

when $\phi_{G}$ is near $-90^{\circ}$. Difficulty in measuring the small value of $\left|E_{43}\right|$ results in error when $\phi_{G}$ is near $90^{\circ}$. Therefore, eq (8) is used to determine the magnitude of $\phi_{G}$ and (9) its quadrant.

\subsection{Measurement of Voltage Ratio}

The circuit shown in figure 2 (b) is used to measure the ratio of the open-circuit voltages of the driving and sensing coils when the calibrator being calibrated is driven by an external vibration exciter. With the switch in the "up" position and with $r_{1}$ set at 10,000 ohms, resistor $r$ is adjusted until the voltages across terminals 1 and 2 and terminals 1 and 3 are equal. The magnitude of the voltage ratio, $R$, is then given by

$$
|R|=\frac{r}{10,000+r} \text {. }
$$

(In the case of a calibrator for which $10,000 \mathrm{ohms}$ is not effectively an infinite impedance across the driving coil, $r_{1}$ in figure 2 (b) should be increased to an adequate value and eq (10) correspondingly modified.)

To determine the phase angle of $R,\left|E_{13}\right|$ and $\left|E_{23}\right|$ are measured. The switch is then put in the "down" position, $r_{1}$ is adjusted until $\left|E_{14}\right|$ equals $\left|E_{13}\right|$, and $\left|E_{43}\right|$ is measured. Then from a construction of a polygon of voltages

and

$$
\phi_{R}=\cos ^{-1}\left(1-\frac{1}{2} \frac{\left|E_{23}^{2}\right|}{\left|E_{13}^{2}\right|}\right),
$$

$$
\phi_{R}=\cos ^{-1}\left(1-\frac{1}{2} \frac{\left|E_{43}^{2}\right|}{\left|E_{13}^{2}\right|}\right)-\cos ^{-1}(|R|) \text {. }
$$

It happens that $\phi_{R}$ is near either $0^{\circ}$ or $180^{\circ}$. Equation (11) is insensitive for these angles, either because $\left|E_{23}\right|$ is very small for $\phi_{R}$ near $0^{\circ}$ or because the cosine is insensitive to small changes in $\left|E_{23}\right|$ near $180^{\circ}$. Therefore, eq (12) is used to determine the magnitude of $\phi_{R}$ and eq (11) only its quadrant.

\section{Typical Results}

\subsection{Calibration of Sensing Coil}

Results obtained in the calibration of the sensing coil of a typical calibrator having a nominal 50-lb driving-force rating are now presented. All measurements were made after thermal equilibrium was approached. The positive terminals of the driving and sensing coils were determined as those having positive voltage when the mounting table had inward velocity. Positive velocity is then outward.

First, the transfer admittance, $G$, experiment 1, was measured for a sequence of weights, $W$, on the mounting table at each frequency for which a calibration was desired. Typical results at frequencies of 900 and $5,000 \mathrm{cps}$ are presented in figure 3. In figure 4 are the corresponding plots of $W /\left(G-G_{0}\right)$ against $W$. The data were fitted by a weighted least-squares procedure for a straight line. The weighting as determined by a consideration of the expected error in $1 /\left(G-G_{o}\right)$ was $1,2,3, \ldots, 10$ for $W=0.1,0.2,0.3, \ldots, 1.0 \mathrm{lb}$. Intercepts $J$ and 

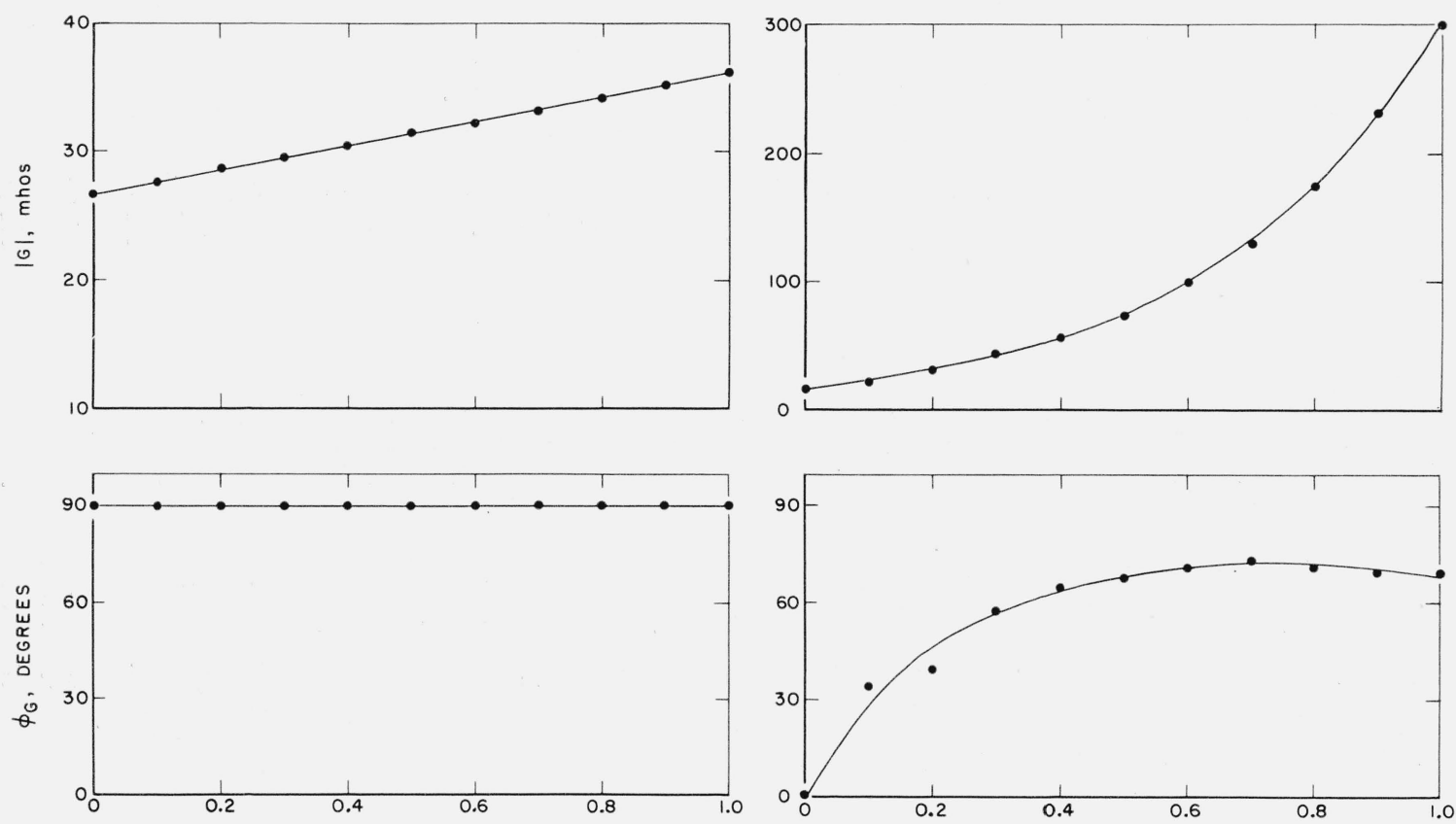

(a)

W, POUNDS

(b)

Figure 3. Variation of transfer admittance, $G$, with weight, $W$, on the mounting table for a typical vibration pickup calibrator at frequencies of (a) $900 \mathrm{cps}$ and (b) 5,000 cps.
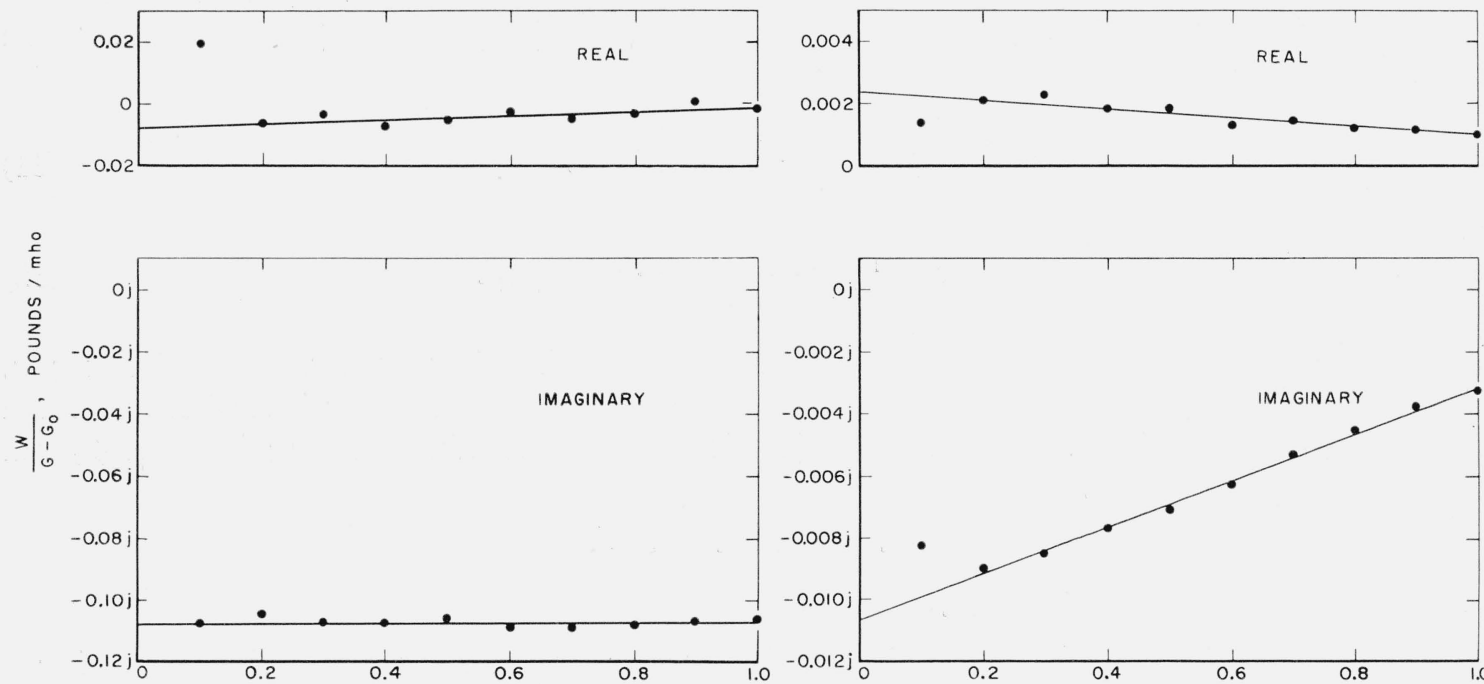

(a)

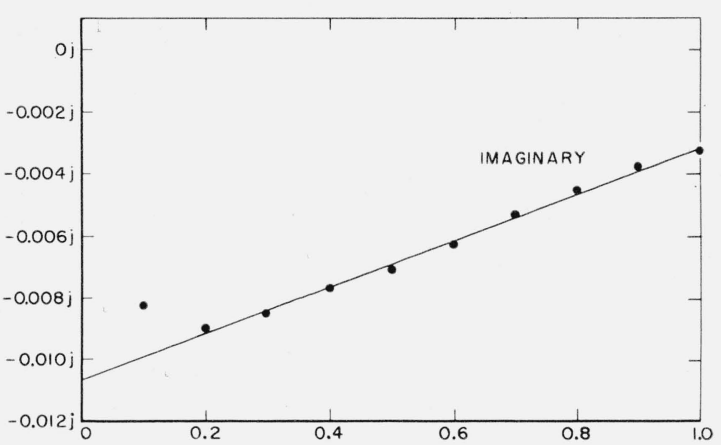

(b)

Figure 4. Plot of $W /\left(G-G_{o}\right)$ as a function of $W$ for a typical vibration pickup calibrator at frequencies of (a) $900 \mathrm{cps}$ and (b) 5,000 cps.

slopes $Q$, determined by this procedure, are at $900 \mathrm{cps} J=0.1089 /-94.2^{\circ} \mathrm{lb}$-ohm, and $Q=$ $0.00767 / 11.8^{\circ} \mathrm{ohm}$, and at $5,000 \mathrm{cps}, J=0.01094 /-77.4^{\circ} \mathrm{lb}-\mathrm{ohm}$, and $Q=0.00765 / 100.4^{\circ} \mathrm{ohm}$.

The voltage ratio, $R$, when driven by an external vibration exciter, experiment 2 , was then determined as $R=0.1090 /-2.6^{\circ}$ at $900 \mathrm{cps}$ and $R=0.2495 /-2.0^{\circ}$ at $5,000 \mathrm{cps}$. Next the constants $a$ and $b$ in eq (4) were computed. Substituting their values in eq (1) gave calibration factors of the sensing coil with a pickup of mechanical impedance $Y_{p}$ on the mounting table, 

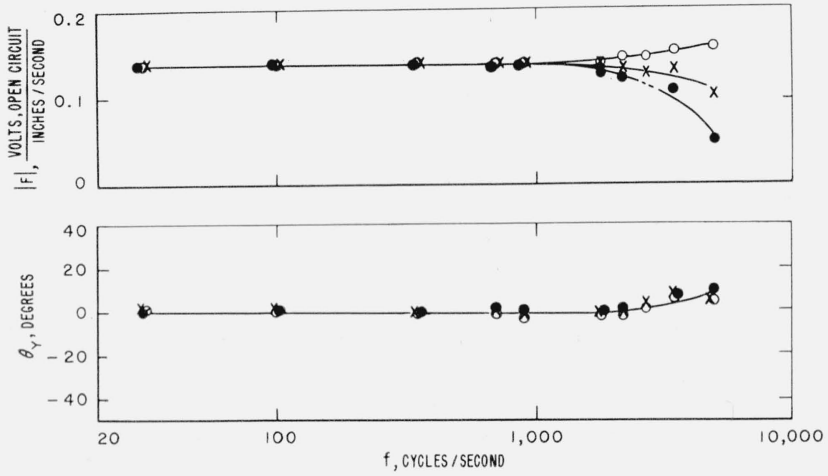

FIgURE 5. Tariation of calibration factor of velocity-sensing coil with frequency and pickup weight for a typical vibration pickup calibrator.

$\mathrm{O}=0 \mathrm{lb}, \times=1 / 2 \mathrm{lb}, \boldsymbol{\bullet}=1 \mathrm{lb}$.

at $900 \mathrm{cps}, F=0.1402 /-3.4^{\circ}+0.000674 / 12.6^{\circ} Y_{p} \mathrm{v}$-sec/in., and at $5,000 \mathrm{cps}, F=0.1584 / 5.5^{\circ}+$ $0.001360 / 93.1^{\circ} Y_{p}$ v-sec/in.

In figure 5 are shown the magnitude and phase angle of the calibration factor of the velocity-sensing coil of this typical vibration-pickup calibrator as a function of frequency for pickups having a mechanical impedance $Y_{p}$ corresponding to weights $W$ of $0,0.5$, and 1.0 lb $\left(Y_{p}=j \omega W / 386\right)$. It is evident that, for this particular vibration-pickup calibrator, the calibration factor of the velocity-sensing coil is nearly independent of frequency and pickup weight up to $900 \mathrm{cps}$. The curve for $1 \mathrm{lb}$ is dotted in the vicinity of $2,700 \mathrm{cps}$ because the plot similar to that shown in figure 4 was not linear beyond $0.5 \mathrm{lb}$. The source of this nonlinearity could not be localized. This nonlinearity has not been found in other calibrators of the same make and model number calibrated at the same frequency.

\subsection{Calibration of a Variable-Resistance-Type Accelerometer and a Piezoelectric-Type Accelerometer}

Results obtained in the calibration of a variable-resistance-type accelerometer and piezoelectric-type accelerometer are now presented. In these calibrations the circuit shown in figure 2 (a) was used with the pickup terminals designated 6 and 7 , in place of velocity-sensingcoil terminals 1 and 3 . The method given in section 3.3 was then used for the measurement of $G_{p}$, the value of $G$ with the pickup attached to the mounting table.

For the piezoelectric pickup, the value of $G_{p}$ obtained at $900 \mathrm{cps}$ was $G_{p}=I_{o}^{D} / E_{o}^{S}=$ $28.51 / 91.8^{\circ}$ mhos, and at 5,000 $\mathrm{cps}, G_{p}=37.9 / 43.6^{\circ}$ mhos. With terminal 6 grounded and terminal 7 replacing terminal 3 , the same procedure gave, at $900 \mathrm{cps}, I_{o}^{D} / E_{p}=16.35 / 2.5^{\circ} \mathrm{mhos}$, and at $5,000 \mathrm{cps}, I_{o}^{D} / E_{p}=3.201 /-44.6^{\circ} \mathrm{mhos}$, where $E_{p}$ is the output voltage of the pickup. From eq (5), the mechanical impedance, $Y_{p}$, of the pickup was computed by using, at 900 cps, $G_{o}=26.81 / 92.0^{\circ} \mathrm{mhos}$, and at $5,000 \mathrm{cps}, G_{o}=16.51 / 18.1^{\circ} \mathrm{mhos}$, and the values of $J$ and $Q$ obtained in section 4.1. This gave, at $900 \mathrm{cps}, Y_{p}=2.712 / 83.3^{\circ} \mathrm{lb}-\mathrm{sec} / \mathrm{in}$. and at $5,000 \mathrm{cps}$, $Y_{p}=18.23 / 70.5^{\circ} \mathrm{lb}$-sec/in. Using these values of $Y_{p}$ in eq (1), the velocity sensing-coil calibration factors at $900 \mathrm{cps}$ and $5,000 \mathrm{cps}$, respectively, are $F=0.1399 /-2.7^{\circ} \mathrm{v}$-sec/in. and $F=$ $0.1357 / 9.2^{\circ} \mathrm{V}$-sec/in. The pickup acceleration calibration factor, $F_{p}$, is

$$
F_{p}=\frac{\left(I_{o}^{D} / E_{o}^{S}\right)}{\left(I_{o}^{D} / E_{p}\right)} \frac{F g}{j \omega}
$$

where $\mathrm{Fg} /(j \omega)$ is the acceleration calibration factor of the sensing coil. Using this equation, at $900 \mathrm{cps}, F_{p}=0.0166 /-3.4^{\circ} \mathrm{v} / \mathrm{g}$, and at $5,000 \mathrm{cps}, F_{p}=0.0197 / 6.5^{\circ} \mathrm{v} / \mathrm{g}$. Figure 6 shows the complete calibration of the piezoelectric-type accelerometer.

The calibration of the variable-resistance-type accelerometer, which was performed in a similar manner, is shown in figure 7. To compute the results on the variable-resistance 

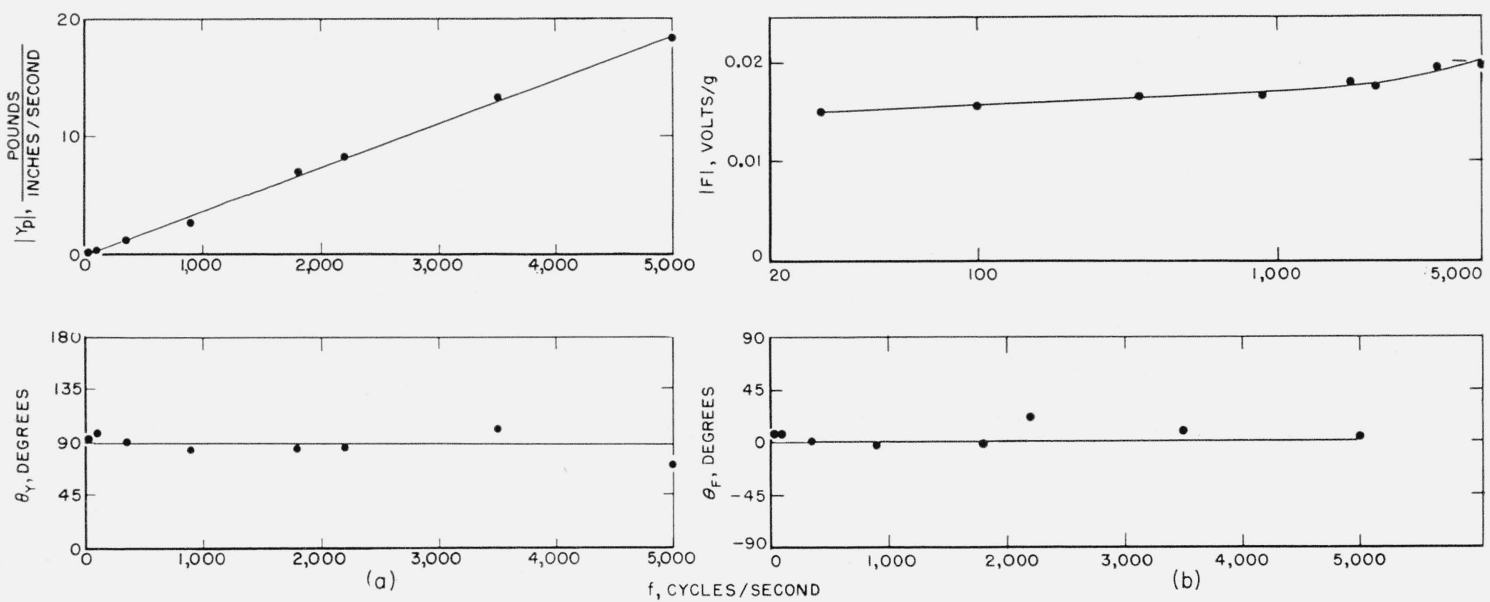

Figure 6. Calibration of piezoelectric-type accelerometer at a nominal acceleration level of $2 \mathrm{~g}$.
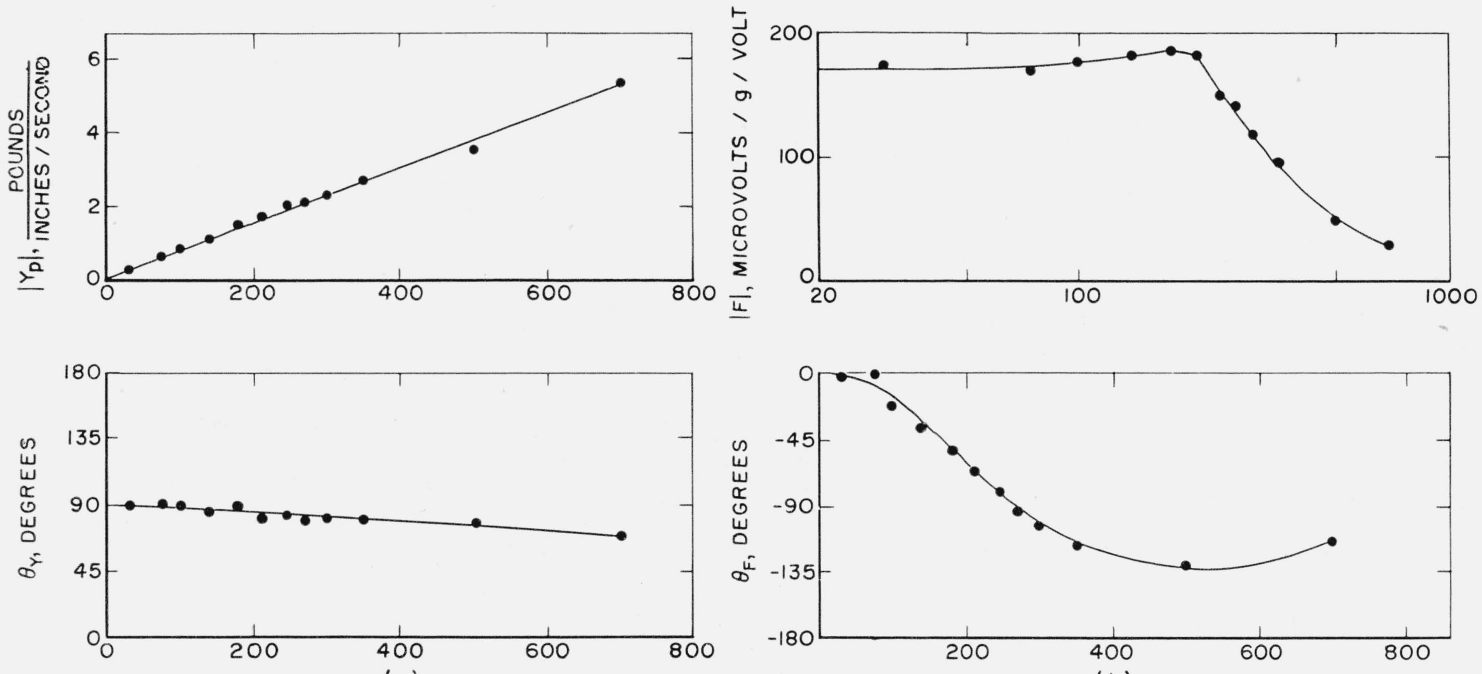

(a)

$f$, CYCLES/SECOND

(b)

Figure 7. Calibration of variable-resistance-type accelerometer at a nominal acceleration level of $2 \mathrm{~g}$.

pickup at frequencies other than those at which the vibration-pickup calibrator was calibrated, it was necessary to interpolate suitably for $a, b, R$, and $Q$, and compute $J$ from eq 4 . This interpolation was only used at frequencies below $900 \mathrm{cps}$. In this frequency range, such interpolation results in no appreciable error for this calibrator when the resonant frequencies of the flexures are avoided. The frequencies used for the pickup calibration were not those at which flexure resonance was present.

\section{Effects of Calibrator Construction on Accuracy}

Inaccuracy in the reciprocity method, exclusive of electrical measurements, arises primarily from deviations from true linearity in the calibrator performance. The more important forms of nonlinearity are discussed below. 


\subsection{Resonance Effects}

Resonance accentuates inaccuracy due to nonlinearity because relatively small changes in the calibrator constants, which are caused by very small changes in the structure of the calibrator and small changes in the exciting frequency, can cause large changes in the amplitude of vibration. Even when such changes do not occur, the large amplitudes associated with resonance may exceed the linear range. The resonance of the driving coil relative to the velocitysensing coil reduces the accuracy of the reciprocity calibration over a small frequency range because $|R|$ is very small and therefore difficult to measure, and because the large amplitude of the driver coil may exceed its linear range. Operation of the calibrator near resonance frequencies should therefore be avoided. Some common resonance conditions in their usual order of appearance with increasing frequency follow:

a. Resonance of mounting table and coils as a rigid body on the guiding flexures.

b. Local resonance in the flexures.

c. Transverse vibration of the shaft connecting mounting table and driving coil.

d. Longitudinal resonance of shaft connecting mounting table and driving coil.

e. Local resonance in table or coils.

\subsection{Transverse Motion}

Transverse motion from any source invalidates the reciprocity method, which requires the mounting table to have uniaxial motion. Transverse motion occurs at the resonance frequencies of the flexures and at the transverse resonance frequencies of the shaft on which the moving parts are mounted. At resonance, the amplitude will vary substantially for small changes in frequency and the resonance may occur at a frequency that is a harmonic of the driving frequency. Flexures can be detuned by attaching small weights to them.

\subsection{Tightness of Mechanical Joints}

The tightness of the mechanical joints in a calibrator will have little effect on its performance at low frequencies, where the moving assembly of mounting table and coils acts as a "rigid body." At frequencies approaching those for internal resonance in the assembly, looseness in the joints can affect behavior by, for example, changing the structural stiffness or by introducing coulomb friction. At very high frequencies, joints normally thought to be tight may be a source of erratic behavior, which is difficult to eliminate as tightness will ordinarily vary with temperature and time. Coating all joints with oil improves their rigidity.

\subsection{Amplitude Effects}

The nonuniformity of the magnetic fields surrounding the coils is a primary source of nonlinearity. This effect tends to be greatest at low frequencies, where the displacement amplitude is large for a given acceleration. At high frequencies, the displacement amplitude is relatively small, and this effect is negligible. The change in equilibrium position of the moving assembly of a calibrator, when its orientation is changed, may cause a change in calibration factor due to change in the effective field strength.

The structure of a calibrator will ordinarily experience small deformations in its linear range at permissible current intensities. At resonance, the deformations are larger. Little is known about the linearity of structural damping for a structure as complicated as a calibrator; however, so long as the damping is small, its possible lack of linearity would affect only the resonant response.

\subsection{Temperature Effects}

Changes in temperature cause moderate changes in elastic constants and in the tightness of joints, and in this way can affect the calibrator performance near resonance. Changes in temperature may also affect the field flux density, and thus the electrical characteristics of the driving coil, but should not ordinarily affect the calibration factor of the velocity-sensing coil. 


\subsection{Purity of Electric-Power Sources}

If the magnets are excited by direct current containing an alternating-current ripple, this ripple may appear in the velocity output of the calibrator owing to currents included in the driving coil by transformer action from the field. Such a disturbance can readily be detected by exciting the field and observing the velocity of the mounting table with the driving coil short-circuited.

Any harmonic content in the power supply to the driving coil will excite the calibrator at the harmonic frequencies, as well as at the primary frequency. The techniques described in this paper are applicable only for excitation at a single frequency.

\section{Conclusions}

It is concluded that the reciprocity methods described can be used for the accurate calibration of vibration pickups and calibrators having linear response. The accuracy is limited primarily by the accuracy of electrical measurement, by deviations from true linearity in construction, and by impurities in power supplies to the field and driving coils. The frequency range is limited theoretically only to frequencies for which the mechanical impedance of the weights attached to the mounting table can be computed. Ordinarily this limits the range to that at which the weights act as though they were rigid bodies. Practically, the calibrator used as an example in this report is most suited for use at frequencies of 900 cps and below, where its calibration factor is not affected by the mechanical impedance of most vibration pickups that would be attached to its mounting table. The calibrator is suited for use above $900 \mathrm{cps}$, however, with some loss in precision primarily due to the electromechanical properties of the calibrator being less constant with respect to field-coil temperature than they are at lower frequencies. The upper acceleration range of most calibrators, when resonance is avoided, is below $50 \mathrm{~g}$. This would be the limit for calibrating by the reciprocity method.

\section{Appendix. General Reciprocity Theory}

\subsection{Reciprocity for Electric Circuit}

If a complicated electric circuit is considered to be made up of a number of meshes, the almost trivial fact that the common impedance between meshes $i$ and $k$, for example, is the same as that between $k$ and $i$ is the basis for the proof of the reciprocity theorem. This proof is elegantly presented by Guillemin on page 152 of reference [6]. Guillemin states on page 276 that the reciprocity theorem can be proved for both transient and steady-state performance. This means, for example, that if we impress a voltage in mesh 5 of a given network and measure the current, say in mesh 2 , and then place the voltage in mesh 2 instead of in mesh 5 and measure the current in mesh 5, we will find the current exactly the same in the two cases, both in magnitude and phase.

\subsection{Reciprocity for Mechanical System}

If a complicated mechanical system can be replaced by an equivalent system having discrete mass-points joined by springs and dashpots, the fact that the spring and dashpot connecting points $i$ and $k$ is the same as that connecting points $k$ and $i$ makes possible a proof of the reciprocity theorem for mechanical systems completely analogous with that for electric circuits. (Reference [7] gives a proof of this theorem for conservative systems.) In other words, this reciprocity theorem states, for example, that if we impress a force on point 5 of a given system and measure the velocity, say at point 2 , we will find the same velocity in magnitude and phase when we place the force at point 2 instead of at point 5 and measure the velocity at point 5 . It should be mentioned that it is possible to have a mechanical system consisting of discrete elements that is not reciprocal, for example, if it contains gyroscopic elements. 


\subsection{Reciprocity for Combined System}

In developing the reciprocity theorem for the combined electric-mechanical system of a calibrator, we will use matrix notation. This will permit us to consider the coils as flexible bodies and take account of electric coupling between coils, as well as deformation in the field and movable structure. The notation and theorems given by Frazer, Duncan, and Collar [8] will be used.

Currents and voltages in the velocity-sensing coil will be indicated by $S$ and in the driving coil by $D$. We will consider the coils subdivided into a sufficient number of segments so each moves as a rigid body and number them consecutively in the two coils. The subscript $o$ will indicate terminal values. Complex notation used in alternating-current electrical theory will be used throughout. All symbols represent vector quantities, unless otherwise noted. An English system of units (inches, pounds, and seconds) will be used.

Current $I_{o}^{S}$ at the terminals of the sensing coil is given by

$$
I_{o}^{S}=\frac{E_{o}^{S}}{z_{o o}^{S S}}+\frac{E_{o}^{D}}{z_{o o}^{S D}}+\left[z^{S}\right]\{E\}
$$

where

$E_{o}^{S}=$ voltage at terminals of coil $S$.

$E_{o}^{D}=$ voltage at terminals of coil $D$.

$z_{o o}^{S S}=$ impedance at terminals of coil $S$.

$z_{o o}^{S D}=$ transfer impedance from terminals of coil $S$ to terminals of coil $D$.

$\left[z^{S}\right]=\left[\frac{1}{z_{o 1}^{S}} \frac{1}{z_{o 2}^{S}} \cdots \frac{1}{z_{o n}^{S}}\right]$, where $z_{o n}^{S}$ is the transfer impedance from segment $n$ to the termi$\{E\}=\left[\begin{array}{c}E_{1} \\ E_{2} \\ \cdot \\ \cdot \\ E_{n}\end{array}\right], E_{n}$ is the voltage generated in segment $n$.

A similar expression can be written for $I_{0}^{D}$. Both of these expressions can be expressed as

where

$$
\left\{I_{o}\right\}=\left[z_{o}\right]\left\{E_{o}\right\}+[z]\{E\},
$$

$$
\begin{aligned}
\left\{I_{o}\right\} & =\left[\begin{array}{c}
I_{o}^{S} \\
I_{o}^{D}
\end{array}\right] ; \quad\left\{E_{o}\right\}=\left[\begin{array}{l}
E_{o}^{S} \\
E_{o}^{D}
\end{array}\right] \\
{\left[z_{0}\right] } & =\left[\begin{array}{cc}
\frac{1}{z_{o o}^{S S}} & \frac{1}{z_{o o}^{S D}} \\
\frac{1}{z_{o o}^{D S}} & \frac{1}{z_{o o}^{D D}}
\end{array}\right] \\
{[z] } & =\left[\begin{array}{cccc}
\frac{1}{z_{o 1}^{S}} & \frac{1}{z_{o 2}^{S}} & \cdots & \frac{1}{z_{o n}^{S}} \\
\frac{1}{z_{o 1}^{D}} & \frac{1}{z_{o 2}^{D}} & \cdots & \frac{1}{z_{o n}^{D}}
\end{array}\right] .
\end{aligned}
$$


The current in any segment is given by

where

$$
\{I\}=[z]^{\prime}\left\{E_{o}\right\}+[Z]\{E\},
$$

$$
\begin{aligned}
\{I\} & =\left[\begin{array}{c}
I_{1} \\
I_{2} \\
\cdot \\
\cdot \\
I_{n}
\end{array}\right], I_{n} \text { is the current in segment } n, \\
{[Z] } & =\left[\begin{array}{cccc}
\frac{1}{z_{11}} & \frac{1}{z_{12}} & \cdots & \frac{1}{z_{1 n}} \\
\frac{1}{z_{21}} & \frac{1}{z_{22}} & \cdots & \frac{1}{z_{2 n}} \\
\cdot & \cdot & & \cdot \\
\cdot & \cdot & & \cdot \\
\cdot & \cdot & & \cdot \\
\frac{1}{z_{m 1}} & \frac{1}{z_{m 2}} & \ldots & \frac{1}{z_{m n}}
\end{array}\right], z_{m n} \text { is the transfer impedance from segment } m \text { to segment } n,
\end{aligned}
$$

$[z]^{\prime}=$ transpose of $[z]$.

Velocity $v_{o}$ at the mounting table, taken positive outward, is given by

$$
v_{0}=\frac{F_{o}}{Y_{o o}}+L_{1}[y][k]\{I\},
$$

where

$F_{0}=$ the force at the mounting table.

$Y_{o o}=$ the mechanical impedance at the mounting table.

$L_{1}=2.249 \times 10^{-7}$, a conversion factor.

$[y]=\left[\begin{array}{llll}\frac{1}{y_{o 1}} & \frac{1}{y_{o 2}} & \cdots & \frac{1}{y_{o n}}\end{array}\right]$, where $y_{o n}$ is the transfer mechanical impedance from the $[k]=\left[\begin{array}{ccccc}B_{1} l_{1} & 0 & 0 & \ldots & 0 \\ 0 & B_{2} l_{2} & 0 & \ldots & 0 \\ 0 & 0 & B_{3} l_{3} & \ldots & 0 \\ \cdot & \cdot & \cdot & & \cdot \\ \cdot & \cdot & \cdot & & \cdot \\ 0 & 0 & 0 & \ldots & B_{n} l_{n}\end{array}\right]$, where $B_{n}$ is the flux density, and $l_{n}$ is the length for 
In eq (A3), the product $L_{1} B_{n} l_{n} I_{n}$ gives the force, in pounds, at segment $n$ when $I_{n}$ is the current, in amperes. The velocity of the various segments is given by

$$
\{v\}=[y]^{\prime} F_{o}+L_{1}[Y][k]\{I\},
$$

where

$$
\begin{aligned}
& \{v\}=\left[\begin{array}{c}
v_{1} \\
v_{2} \\
\cdot \\
\cdot \\
v_{n}
\end{array}\right], v_{n} \text { is the velocity of segment } n . \\
& {[y]^{\prime}=\text { transpose of }[y] .}
\end{aligned}
$$

$$
[Y]=\left[\begin{array}{cccc}
\frac{1}{y_{11}} & \frac{1}{y_{12}} & \cdots & \frac{1}{y_{1 n}} \\
\frac{1}{y_{21}} & \frac{1}{y_{22}} & \cdots & \frac{1}{y_{2 n}} \\
\cdot & \cdot & & \cdot \\
\cdot & \cdot & & \cdot \\
\cdot & \cdot & & \cdot \\
\frac{1}{y_{m 1}} & \frac{1}{y_{m 2}} & \cdots & \frac{1}{y_{m n}}
\end{array}\right], \begin{aligned}
& y_{m n} \text { is the transfer mechanical impedance from segment } m \text { to } \\
& \text { segment }
\end{aligned}
$$

The corresponding velocity in the magnetic field at the various segments is given by

$$
\left\{v^{M}\right\}=L_{1}\left[Y^{M}\right][k]\{I\}+\left[y^{M}\right]^{\prime} F_{o},
$$

where

$\left\{v^{M}\right\}=\left[\begin{array}{c}v_{1}^{M} \\ v_{2}^{M} \\ \cdot \\ \cdot \\ v_{n}^{M}\end{array}\right], v_{n}^{M}$ is the velocity of the magnetic field at segment $n$.

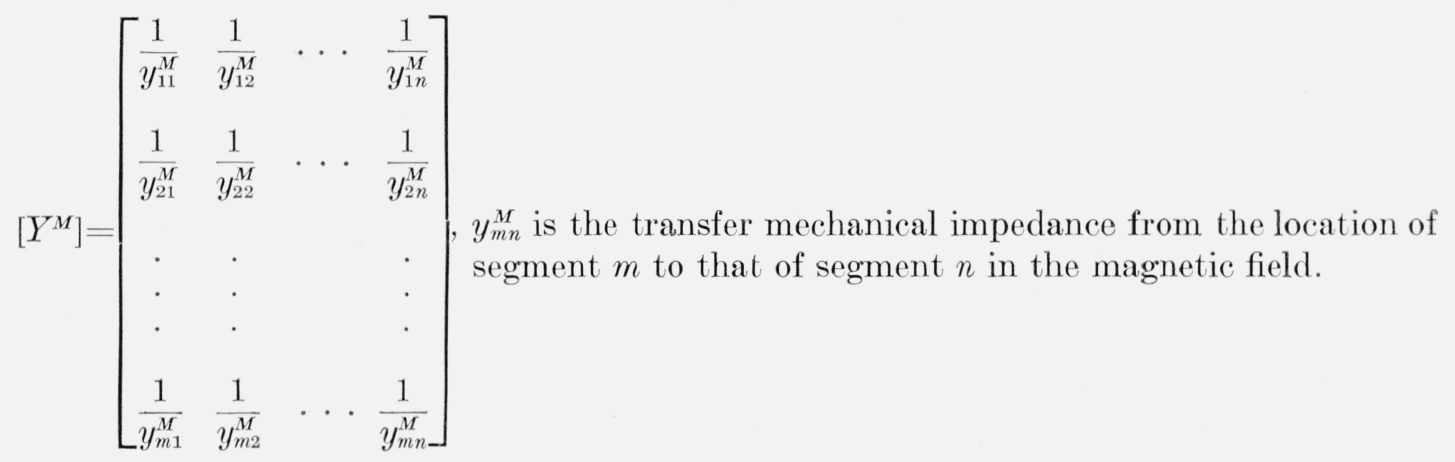




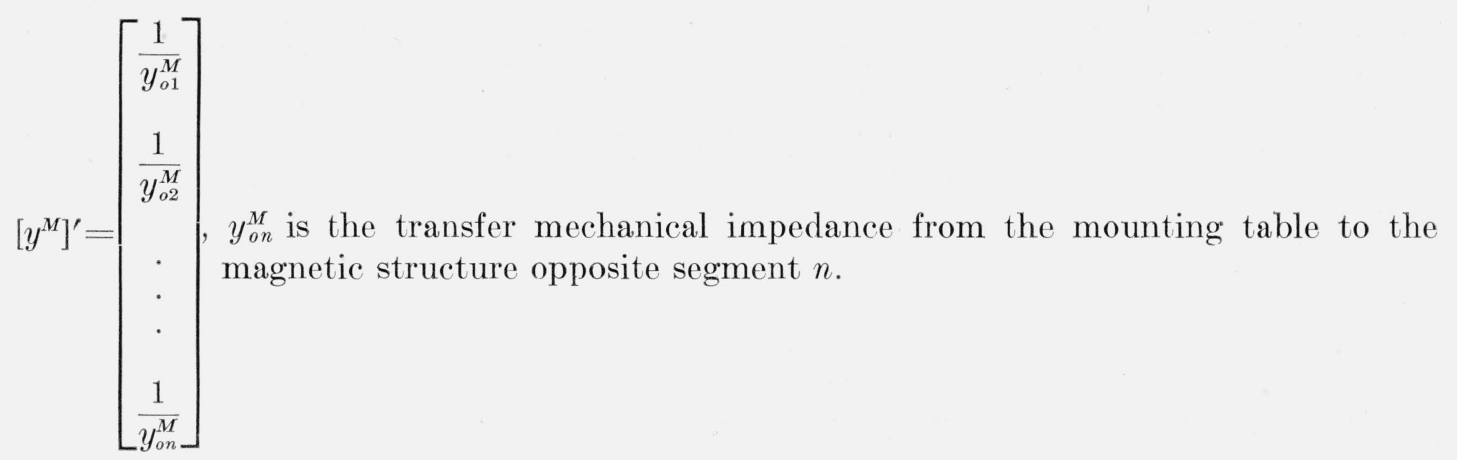

The voltage generated in each segment is

$$
\{\boldsymbol{E}\}=-L_{2}[k]\{v\}+L_{2}[k]\left\{v^{M}\right\},
$$

where $L_{2}=2.540 \times 10^{-8}$, a conversion factor. In eq $(\mathrm{A} 5),-L_{2} B_{n} l_{n}\left(v_{n}-v_{n}^{M}\right)$ is the electromotive force generated in segment $n$, in volts, when the relative velocity between segment $n$ and the magnetic field, in inches per second, is $\left(v_{n}-v_{n}^{M}\right)$. Substituting (A2) into (A4) and (A4a), and substituting the results into (A5) gives

$$
\{\boldsymbol{E}\}=-F_{0} L_{2}[k]\left[y-y^{M}\right]^{\prime}-L_{1} L_{2}[K][z]^{\prime}\left\{E_{o}\right\}-L_{1} L_{2}[K][Z]\{E\},
$$

where

$$
[K]=[k][Y][k]-[k]\left[Y^{M}\right][k] .
$$

Letting

$$
\left[V^{-1}\right]=[1]+L_{1} L_{2}[K][Z],
$$

where

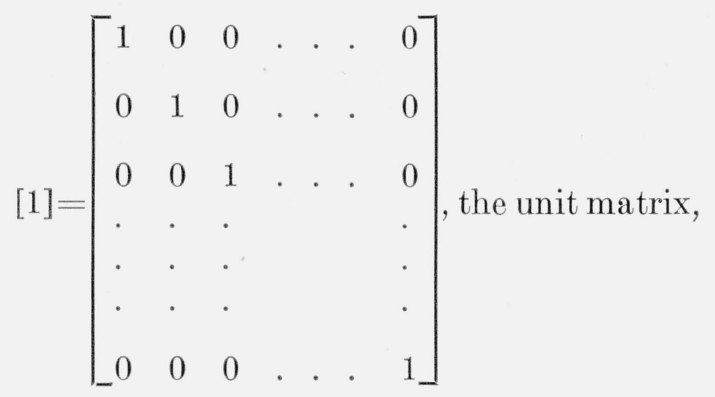

$\left[V^{-1}\right]=$ inverse of $[V]$.

$$
\{E\}=-F_{o} L_{2}[V][k]\left[y-y^{M}\right]^{\prime}-L_{1} L_{2}[V][K][z]^{\prime}\left\{E_{o}\right\} .
$$

Substituting (A9) into (A1a),

$$
\left\{I_{o}\right\}=\left[z_{0}\right]\left\{E_{o}\right\}-F_{o} L_{2}[z][V][k]\left[y-y^{M}\right]^{\prime}-L_{1} L_{2}[z][V][K][z]^{\prime}\left\{E_{o}\right\} .
$$

Substituting (A9) in (A2) and the result in (A3) gives

$$
v_{o}=\frac{F_{o}}{Y_{o o}}-L_{1} L_{2} F_{o}\left[y-y^{M}\right][k][Z][V][k]\left[y-y^{M}\right]^{\prime}+L_{1}\left[y-y^{M}\right][k][V]^{\prime}[z]^{\prime}\left\{\boldsymbol{E}_{o}\right\},
$$

using

$$
[V]^{\prime}=[1]-L_{1} L_{2}[Z][V][K] .
$$


The proof of eq (A12) is obtained as follows: By using (A8),

$$
\begin{aligned}
{[1]-L_{1} L_{2}[Z][V][K] } & =\left[K^{-1}\right]\left([K]-L_{1} L_{2}[K][Z][V][K]\right) \\
& =\left[K^{-1}\right]\left([K]+\left(1-\left[V^{-1}\right]\right)[V][K]\right) \\
& =\left[K^{-1}\right][V][K] .
\end{aligned}
$$

Also, premultiplying both sides of (A8) by $\left[K^{-1}\right]$,

$$
\left[K^{-1}\right]\left[V^{-1}\right]=\left[K^{-1}\right]+L_{1} L_{2}[Z] .
$$

As $[K]$ is symmetric, $\left[K^{-1}\right]$ is symmetric. As $[Z]$ is also symmetric, $\left[K^{-1}\right]\left[V^{-1}\right]$ is symmetric. Its inverse $[V][K]$ is therefore also symmetric. The transpose of a symmetric matrix is itself or

$$
[V][K]=[K]^{\prime}[V]^{\prime}=[K][V]^{\prime} .
$$

Substituting (A15) in (A13) gives (A12).

The current in the velocity-sensing coil is always zero,

$$
I_{o}^{S}=\left[\begin{array}{ll}
1 & 0
\end{array}\right]\left\{I_{o}\right\}=0 .
$$

Substituting (A10) into (A16) and solving for $E_{o}^{S}$ gives

$$
E_{o}^{S}=\frac{F_{o} L_{2} z_{o o}^{S S}\left[z^{S}\right][V][k]\left[y-y^{M}\right]^{\prime}-E_{o}^{D}\left(\frac{z_{o o}^{S S}}{\left.z_{o o}^{S D}-L_{1} L_{2} z_{o o}^{S S}\left[z^{S}\right][V][K]\left[z^{D}\right]^{\prime}\right)}\right.}{1-L_{1} L_{2} z_{o o}^{S S}\left[z^{S}\right][V][K]\left[z^{S}\right]^{\prime}} .
$$

The current in the driving coil is

$$
I_{o}^{D}=\left[\begin{array}{ll}
0 & 1
\end{array}\right]\left\{I_{o}\right\}
$$

Substituting (A17) into (A10) and the result into (A18) gives

$$
\begin{gathered}
\left(\frac{z_{o D}^{S S}}{z_{o o}^{S D}}\left[z^{S}\right][V][k]\left[y-y^{M}\right]^{\prime}-\left[z^{D}\right][V][k]\left[y-y^{M}\right]^{\prime}\right. \\
I_{o}^{D}=F_{o} L_{2} \frac{\left.+L_{1} L_{2} z_{o o}^{S S}\left[z^{S}\right][V][K]\left[z^{S}\right]^{\prime}\left[z^{D}\right][V][k]\left[y-y^{M}\right]^{\prime}-L_{1} L_{2} z_{o o}^{S S}\left[z^{S}\right][V][K]\left[z^{D}\right]^{\prime}\left[z^{S}\right][V][k]\left[y-y^{M}\right]^{\prime}\right)}{1-L_{1} L_{2} z_{o o}^{S S}\left[z^{S}\right][V][K]\left[z^{S}\right]^{\prime}} \\
\left(\frac{1}{z_{o o}^{D D}-\frac{z_{o o}^{S S}}{\left(z_{o o}^{S D}\right)^{2}}-L_{1} L_{2} \frac{z_{o o}^{S S}}{z_{o o}^{D D}}\left[z^{S}\right][V][K]\left[z^{S}\right]^{\prime}+2 L_{1} L_{2} \frac{z_{o o}^{S S}}{z_{o o}^{S D}}\left[z^{S}\right][V][K]\left[z^{D}\right]^{\prime}-L_{1} L_{2}\left[z^{D}\right][V][K]\left[z^{D}\right]^{\prime}}\right. \\
\left.+L_{1}^{2} L_{2}^{2} z_{o o}^{S S}\left[z^{S}\right][V][K]\left(\left[z^{S}\right]^{\prime}\left[z^{D}\right]-\left[z^{D}\right]^{\prime}\left[z^{S}\right]\right)[V][K]\left[z^{D}\right]^{\prime}\right) \\
+E_{o}^{D}-
\end{gathered}
$$

Substituting (A17) into (A11) gives

$$
\begin{aligned}
& \left(\frac{1}{Y_{o o}}-L_{1} L_{2}\left[y-y^{M}\right][k][Z][V][k]\left[y-y^{M}\right]^{\prime}-L_{1} L_{2} \frac{z_{o o}^{S S}}{Y_{o o}}\left[z^{S}\right][V][K]\left[z^{S}\right]^{\prime}\right. \\
& +L_{1}^{2} L_{2}^{2} z_{o o}^{S S}\left[z^{S}\right][V][K]\left[z^{S}\right]^{\prime}\left[y-y^{M}\right][k][Z][V][k]\left[y-y^{M}\right]^{\prime} \\
& \left.+L_{1} L_{2} z_{o o}^{s S}\left[z^{s}\right][V][k]\left[y-y^{M}\right]^{\prime}\left[z^{s}\right][V][k]\left[y-y^{M}\right]^{\prime}\right) \\
& v_{o}=F_{o} \frac{+L_{1} L_{2} z_{o o}^{S S}\left[z^{S}\right][V][K]\left[z^{S}\right]^{\prime}}{1-L_{1} L_{2} z_{o o}} \\
& \left(\frac{z_{o o}^{S S}}{z_{o o}^{S D}}\left[z^{S}\right][V][k]\left[y-y^{M}\right]^{\prime}-\left[z^{D}\right][V][k]\left[y-y^{M}\right]^{\prime}\right. \\
& -L_{1} E_{o}^{D} \frac{\left.+L_{1} L_{2} z_{o o}^{S S}\left[z^{S}\right][V][K]\left[z^{S}\right]^{\prime}\left[z^{D}\right][V][k]\left[y-y^{M}\right]^{\prime}-L_{1} L_{2} z_{o o}^{s o}\left[z^{S}\right][V][K]\left[z^{D}\right]^{\prime}\left[z^{S}\right][V][k]\left[y-y^{M}\right]^{\prime}\right)}{1-L_{1} L_{2} z_{o o}^{S S}\left[z^{S}\right][V][K]\left[z^{S}\right]^{\prime}} .
\end{aligned}
$$


The coefficient of $L_{2} F_{o}$ in equation (A19) equals the coefficient of $-L_{1} E_{o}^{D}$ in (A20). Equations (A19) and (A20) are subjected first to the condition $v_{o}=0$ and then to the condition $I_{o}^{D}=0$. Eliminating $E_{o}^{D}$ in $(\mathrm{A} 19)$, when $v_{o}=0$, and $F_{o}$ in $(\mathrm{A} 20)$, when $I_{o}^{D}=0$, we obtain

$$
\left.\frac{F_{o}}{L_{1} I_{o}^{D}}\right|_{v_{o}=0}=-\left.\frac{E_{o}^{D}}{L_{2} v_{o}}\right|_{I_{o}^{D}=0}
$$

This is the reciprocity relation that exists in the combined electromechanical system.

Equations (A20), (A19), and (A17) can be written for brevity as

$$
\begin{gathered}
v_{o}=C F_{o}+A L_{1} E_{o}^{D} \\
I_{o}^{D}=-A L_{2} F_{o}+B L_{1} E_{o}^{D} \\
E_{o}^{S}=H F_{o}+N L_{1} E_{o}^{D},
\end{gathered}
$$

where the constants $A, B, C, H$, and $N$ depend only on the calibrator construction.

In eq (1) a relationship is given for the calibration factor, defined as $F=E_{o}^{S} / v_{o}$, as a function of the mechanical impedance, $Y_{p}$, of the object on the mounting table. We will determine this equation in terms of the calibrator constants $A, B, C, H$, and $N$ of eq (A22) to (A24). If the force, $F_{o}$, is only the reaction to driving a mechanical impedance $Y$ at a velocity $v_{o}$,

$$
v_{o}=-F_{o} / Y .
$$

Substituting (A25) in (A22) and solving for $F_{o}$,

$$
F_{o}=-E_{o}^{D} \frac{A L_{1}}{C+1 / Y}
$$

Substituting (A26) into (A24) and (A25) and forming the ratio $E_{o}^{S} / v_{0}$,

$$
F=\frac{E_{o}^{S}}{v_{o}}=\frac{N}{A}+\left(\frac{N C}{A}-H\right) Y .
$$

We see that $N / A$ in eq (A27) is $a$ in eq (1) and $(N C / A)-H$ in eq (A27) is $b$ in eq (1) when $Y$ is $Y_{p}$.

$$
a=N / A, \quad b=(N C / A)-H .
$$

In experiment 1 , section 2 , the transfer admittance $G=I_{o}^{D} / E_{o}^{S}$ is determined for a series of weights, $W$, attached to the mounting table. Substituting (A26) into (A23) and (A24) and forming the ratio $G$,

$$
G=\frac{I_{o}^{D}}{E_{o}^{S}}=\frac{B+\left(B C+A^{2} L_{2}\right) Y}{N+(N C-A H) Y} .
$$

In experiment $1, Y$ is the mechanical impedance $j \omega W / g$, where $W$ is the weight on the mounting table, $\omega$ the frequency in radians per second, and $g$ the acceleration of gravity, $386 \mathrm{in} . / \mathrm{sec}^{2}$. Making this substitution in (A29) and forming $W /\left(G-G_{0}\right)$, where $G_{o}$ is the value of $G$ when $Y=0$, we find

$$
\frac{W}{G-G_{o}}=\frac{386}{j \omega} \frac{N^{2}}{A^{2} N L_{2}+A B H}+\frac{N^{2} C-A H N}{A^{2} N L_{2}+A B H} W .
$$


We see that the intercept $J$ and slope $Q$ found in determining $W\left(G-G_{o}\right)$ experimentally are

$$
\begin{gathered}
J=\frac{386}{j \omega} \frac{N^{2}}{A^{2} N L_{2}+A B H} \\
Q=\frac{N^{2} C-A H N}{A^{2} N L_{2}+A B H} .
\end{gathered}
$$

In experiment 2, section 2, the voltage ratio, $R=E_{o}^{S} / E_{o}^{D}$, is determined when $I_{o}^{D}$ is zero. Eliminating $F_{o}$ between (A23) and (A24) and setting $I_{o}^{D}=0$, we see that

$$
R=E_{o}^{S} / E_{o}^{D}=\frac{H B L_{1}+A N L_{1} L_{2}}{A L_{2}}
$$

Equation (4) is obtained when eq (A31) through (A33) are substituted into (A28) and $\mathrm{L}_{1}$ and $L_{2}$ have the values given previously.

From eq (A29), $G_{0}=B / N$, when $Y$ is zero, that is, $G_{o}$ is the value of $G$ when nothing is attached to the mounting table of the calibrator. If $G_{p}$ is the value of eq (A29) when $Y=Y_{p}$, where $Y_{p}$ is the mechanical impedance of a pickup to be determined, we find that

$$
Y_{p}=\frac{N^{2}\left(G_{p}-G_{o}\right)}{\left(A^{2} L_{2} N+A H B\right)-\left(N^{2} C-A H N\right)\left(G_{p}-G_{o}\right)} \cdot
$$

The substitution of eq (A31) and (A32) into (A34) gives eq (5) for determining the mechanical impedance of a pickup attached to the mounting table of the calibrator.

We are indebted to L. R. Sweetman for guidance in setting up the laboratory equipment, and to Richard Harwell, Jr., for the careful machining of the masses and the various fixtures used. Ruth Woolley performed the least-squares calculations and Carol Waldron prepared the figures.

\section{References}

[1] Richard K. Cook, Absolute pressure calibrations of microphones, J. Research NBS 25, 489-505 (1940) RP1341.

[2] Horace M. Trent, The absolute calibration of electromechanical pickups, J. Appl. Mechanics 15, 49-52 (1948).

[3] Albert London, The absolute calibration of vibration pickups, NBS Tech. News Bul. 32, 8-10 (1948).

[4] Sanford P. Thompson, Reciprocity calibration of primary vibration standards, Naval Research Laboratory Rept. F-3337, 8 p. (August 16, 1948).

[5] M. Harrison, A. O. Sykes, and P. G. Marcotte, The reciprocity calibration of piezoelectric accelerometers, David Taylor Model Basin Rept. 811, 14 p. (March 1952).

[6] Ernst A. Guillemin, Communication networks (John Wiley \& Sons, Inc., New York, N. Y., 1931).

[7] Horace Lamb, On reciprocal theorems in dynamics, Proc. London Math. Soc. 19, 144-151 (1889).

[8] R. A. Frazer, W. J. Duncan, and A. R. Collar, Elementary matrices (MacMillan Co., New York, N. Y. 1947).

Washington, March 12, 1956. 